\title{
RECONSTRUCTION OF ROUND VOIDS IN THE ELASTIC HALF-SPACE: ANTIPLANE PROBLEM
}

\author{
MEZHLUM A. SUMBATYAN, VINCENZO TIBULLO, AND VITTORIO ZAMPOLI
}

Received 15 February 2005; Revised 24 May 2005; Accepted 12 July 2005

We study the reconstruction of geometry (position and size) of round voids located in the elastic half-space, in frames of antiplane two-dimensional problem. We assume that a known point force is applied to the boundary surface of the half-space, and we can measure the shape of the surface over a certain finite-length interval. Then, if the geometry of the defect is unknown, we construct an algorithm to restore its position and size. Some numerical examples demonstrate a good stability of the proposed algorithm.

Copyright (C 2006 Mezhlum A. Sumbatyan et al. This is an open access article distributed under the Creative Commons Attribution License, which permits unrestricted use, distribution, and reproduction in any medium, provided the original work is properly cited.

\section{Introduction}

In the engineering applications of strength theory, the detection and recognition of defects in elastic materials is one of the most important problems of nondestructive evaluation. Various methods are used for this purpose, and one of them is founded on the theory of inverse problems. In order to detect and recognize the image of the void, one may apply over a boundary of the sample a certain type of load, in order to measure the boundary deformation caused by this load. Then, one may suppose that the presence (or absence) of interior defects will influence the measured obtained data. It is also quite natural to suppose that if there is an interior void in the sample, then its position and geometry can influence significantly the shape of the deformed boundary. This idea creates a good basis for defects reconstruction from the measured deformation of the boundary of loaded samples.

A number of theoretical works were devoted to the inverse problems of this kind, with applications to the recognition of cracks $[1,5,8]$. Some important papers concern the uniqueness of the solution, others develop explicit-form analytical results or numerical algorithms [2,3]. Unfortunately, much less results are devoted to reconstruction of volumetric (nonthin) defects in elastic samples under the same conditions and with the same type of input data. 
In the present work, we study the antiplane (i.e., scalar) problem of linear isotropic elasticity in the half-space, with an outer load applied to its boundary surface. We show that the so-formulated direct problem can be reduced to the Laplace partial differential equation. Then, we construct Green's function, which automatically satisfies the trivial boundary condition over the plane surface of the considered half-space. Such Green's function allows us to formulate the direct problem as a single integral equation holding over the boundary of the void, in the case when a volumetric defect is located in the elastic half-space. Solution of this integral equation permits to determine the shape of the boundary surface, if the form of the void is known. Further, we formulate the inverse problem to restore the geometry of the void from the measured input data, which is the known deformation of the boundary line over some finite-length interval. A specially proposed numerical algorithm is suitable to solve this inverse problem. This is reduced to a sort of minimization of the discrepancy functional. Finally, we give some examples of the application of the proposed method, in the case of the reconstruction (location and size) of round voids.

\section{Mathematical formulation, the basic BIE, and Green's function for the half-space}

Let us consider the (two-dimensional) antiplane problem about a volumetric flaw with the boundary $L$ located in the homogeneous and isotropic elastic half-space $y \geq 0$ (see Figure 2.1). The antiplane formulation implies that the components of the displacement vector $\mathbf{u}$ are of the following form:

$$
\mathbf{u}(x, y, z)=\{0,0, w(x, y)\}
$$

where $w$ is the component of the displacement vector in the direction $z$. Then, the equation of equilibrium can be simplified to the ordinary Laplace equation (see, e.g., [4])

$$
\mu \Delta \mathbf{u}+(\lambda+\mu) \operatorname{grad} \operatorname{div} \mathbf{u}=0 \Longrightarrow \frac{\partial^{2} w}{\partial x^{2}}+\frac{\partial^{2} w}{\partial y^{2}}=0
$$

where $\lambda$ and $\mu$ are elastic constants. It is obvious that the only nontrivial components of the elastic stress tensor, among other six ones, are $\sigma_{x z}$ and $\sigma_{y z}$, which can be represented in terms of function $w(x, y)$ as follows: $\sigma_{x z}(x, y)=\mu \partial w / \partial x, \sigma_{y z}(x, y)=\mu \partial w / \partial y$.

In the direct problem, the position and the geometry of the void are known, but in the inverse problem, they should be determined from some input data. In order to provide such input data, let us assume that a known tangential point force $\sigma_{y z}=\sigma_{0} \delta(x)$ is applied to the boundary line $y=0$ of the half-space, for example, at the origin (see Figure 2.1). Due to linearity of the problem, let us represent the full solution of this problem as a superposition of the two ones: (1) corresponding to the applied load for the fully continuous (i.e., without any void) half-space; (2) corresponding to the problem with the free boundary line $y=0$ and with the void located in the half-space, whose boundary $L$ is subjected to some tangential stress:

$$
w(x, y)=\varphi^{0}(x, y)+\varphi(x, y) .
$$


Mezhlum A. Sumbatyan et al. 3

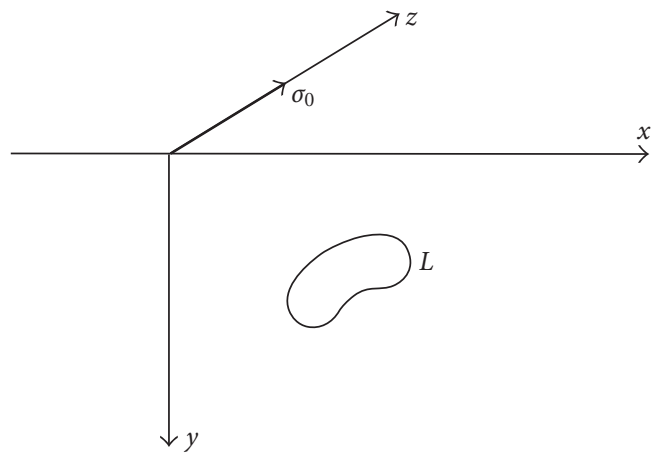

Figure 2.1. Volumetric flaw in the elastic half-space: antiplane problem.

We imply that both functions $\varphi^{0}$ and $\varphi$ satisfy the Laplace equation (2.2). The solution to the problem in the perfect half-plane, free of void and loaded by an outer point force $\sigma_{0}$ at the origin (see Figure 2.1), can be found in classical books on the elasticity theory (see, e.g., [4]):

$$
\varphi^{0}(x, y)=-\frac{\sigma_{0}}{2 \pi \mu} \ln \left(x^{2}+y^{2}\right)
$$

Now we are ready to consider the half-space with void. Let a void with the boundary $L$ free of load be located in the elastic half-space under consideration (see Figure 2.1). Under conditions of the antiplane problem, among all three components of the stress vector on the boundary curve $L$, there is the only one, which reads as

$$
T_{z}=\sigma_{x z} n_{x}+\sigma_{y z} n_{y}=\mu\left(n_{x} \frac{\partial w}{\partial x}+n_{y} \frac{\partial w}{\partial y}\right)=\mu \frac{\partial w}{\partial n}
$$

where $\mathbf{n}$ is the normal to $L$. If the upper plane surface $y=0$ is loaded at the origin by a point force $\sigma_{0}$, then the mathematical formulation of the direct problem is to solve the Laplace equation (2.2), with the boundary conditions

$$
\frac{\partial w}{\partial y}(y=0)=\frac{\sigma_{0}}{\mu} \delta(x),\left.\quad \frac{\partial w}{\partial n}\right|_{L}=0 .
$$

Then, taking into account representation (2.3) and boundary condition (2.7) for function $\varphi^{0}(x, y)$, we can conclude that the boundary value problem for function $\varphi(x, y)$ is formulated as follows:

$$
\frac{\partial^{2} \varphi(x, y)}{\partial x^{2}}+\frac{\partial^{2} \varphi(x, y)}{\partial y^{2}}=0, \quad \frac{\partial \varphi}{\partial y}(y=0)=0,\left.\quad \frac{\partial \varphi}{\partial n}\right|_{L}=-\left.\frac{\partial \varphi^{0}}{\partial n}\right|_{L} .
$$


4 Reconstruction of round voids in the elastic half-space

Let $\Phi(\xi, \eta, x, y)$ denote Green's function for the Laplacian in the considered half-space, that is, the function which satisfies the equation

$$
\frac{\partial^{2} \Phi}{\partial \xi^{2}}+\frac{\partial^{2} \Phi}{\partial \eta^{2}}=-\delta(\xi-x) \delta(\eta-y), \quad y>0, \eta \geq 0,
$$

in the half-space $\eta \geq 0$ with the trivial boundary condition

$$
\frac{\partial \Phi}{\partial \eta}=0, \quad \eta=0
$$

Then, following the classical potential theory [10], we can write out the basic integral representation for the unknown function

$$
\varphi(x, y)=\int_{L}\left(\varphi \frac{\partial \Phi}{\partial n}-\Phi \frac{\partial \varphi}{\partial n}\right) d l=\int_{L}\left(\varphi \frac{\partial \Phi}{\partial n}+\Phi \frac{\partial \varphi^{0}}{\partial n}\right) d l
$$

where we have taken into account the boundary condition (2.7).

Now, applying the limit $(x, y) \rightarrow(X, Y),(X, Y) \in L$, and using the well-known properties of the potential of double layer [10], we can reduce the direct problem to a boundary integral equation (BIE):

$$
\begin{gathered}
\frac{1}{2} \varphi(X, Y)-\int_{L} \varphi(\xi, \eta) \frac{\partial \Phi}{\partial n}(\xi, \eta, X, Y) d l=f(X, Y), \\
f(X, Y)=\int_{L} \frac{\partial \varphi^{0}}{\partial n}(\xi, \eta) \Phi(\xi, \eta, X, Y) d l .
\end{gathered}
$$

Here Green's function $\Phi(\xi, \eta, X, Y)$ should be constructed as a solution to $(2.8)$ with boundary condition (2.9) that is given by the virtual images method in the following form:

$$
\Phi=-\frac{1}{4 \pi}\left\{\ln \left[(x-\xi)^{2}+(y-\eta)^{2}\right]+\ln \left[(x-\xi)^{2}+(y+\eta)^{2}\right]\right\} .
$$

Let us show that BIE (2.11) can be rewritten in a more advanced form, when its righthand side $f(X, Y)$ is free of any integration. For this aim, by using Green's integral formula applied to the pair of functions $\varphi^{0}(\xi, \eta)$ and $\Phi(\xi, \eta, x, y)$ inside the contour $L$, we prove that

$$
\int_{L}\left(\varphi^{0} \frac{\partial \Phi}{\partial n}-\Phi \frac{\partial \varphi^{0}}{\partial n}\right) d l=0
$$

Then, the summation of (2.13) with the left equality in (2.10) gives

$$
\varphi(x, y)=\int_{L}\left(w \frac{\partial \Phi}{\partial n}-\Phi \frac{\partial w}{\partial n}\right) d l=\int_{L} w(\xi, \eta) \frac{\partial \Phi}{\partial n}(\xi, \eta, x, y) d l
$$

due to boundary condition (2.6). It should be noted that both the normal $\mathbf{n}$ and the elementary arc-length $d l$ are applied at the point $(\xi, \eta)$. So, with $(x, y) \rightarrow(X, Y)$, we come 
to a considerably simpler form of the basic BIE than the one written in (2.11):

$$
\frac{w(X, Y)}{2}-\int_{L} w(\xi, \eta) \frac{\partial \Phi}{\partial n}(\xi, \eta, x, y) d l=\varphi^{0}(X, Y), \quad(X, Y) \in L .
$$

A more detailed representation for the kernel $\Phi$ in (2.15) can also be given in a finite form:

$$
\begin{aligned}
\frac{\partial \Phi}{\partial n}=\frac{\partial \Phi}{\partial \xi} & n_{\xi}+\frac{\partial \Phi}{\partial \eta} n_{\eta} \\
= & \frac{1}{2 \pi}\left\{n_{\xi}\left[\frac{x-\xi}{(x-\xi)^{2}+(\eta+y)^{2}}+\frac{x-\xi}{(x-\xi)^{2}+(\eta-y)^{2}}\right]\right. \\
& \left.\quad-n_{\eta}\left[\frac{\eta+y}{(x-\xi)^{2}+(\eta+y)^{2}}+\frac{\eta-y}{(x-\xi)^{2}+(\eta-y)^{2}}\right]\right\}
\end{aligned}
$$

where $n_{\xi}, n_{\eta}$ are direction cosines of the vector $\mathbf{n}$.

\section{Calculation of physical quantities and formulation of the reconstruction problem}

As soon as integral equation (2.15) is solved, that is, the function $w(x, y)$ is determined, the displacement field at arbitrary point of the elastic medium can be calculated by using (2.3), (2.14):

$$
w(x, y)=\varphi^{0}(x, y)+\int_{L} w(\xi, \eta) \frac{\partial \Phi}{\partial n} d l=-\frac{\sigma_{0}}{2 \pi \mu} \ln \left(x^{2}+y^{2}\right)+\int_{L} w(\xi, \eta) \frac{\partial \Phi}{\partial n} d l,
$$

where the quantity $\partial \Phi / \partial n$ is given again by (2.16). After that the components of the stress tensor can be calculated as $\sigma_{x z}=\mu \partial w / \partial x, \sigma_{y z}=\mu \partial w / \partial y$ (see Section 2). We thus can calculate all physical quantities at the arbitrary point $(x, y)$ inside the medium. In particular, the shape of the upper boundary surface $(y=0)$ is

$$
F(x)=-\frac{\sigma_{0}}{\pi \mu} \ln |x|+\frac{1}{\pi} \int_{L} \frac{(x-\xi) n_{\xi}-\eta n_{\eta}}{(x-\xi)^{2}+\eta^{2}} w(\xi, \eta) d l .
$$

From this formula we can easily extract the contribution given by two physically different components: (1) the deformation of the boundary in the perfect (i.e., free of any void) half-space under the applied force $\sigma_{0}$ that is given by the first logarithmic term in (3.2); (2) the contribution given by the influence of presence of the flaw, the second integral term in (3.2). The latter can be calculated as

$$
F_{0}(x)=\frac{1}{\pi} \int_{L} \frac{(x-\xi) n_{\xi}-\eta n_{\eta}}{(x-\xi)^{2}+\eta^{2}} w(\xi, \eta) d l,
$$

and gives, as has been said above, the contribution of the defect presence to the deformation of the boundary surface.

Concrete realization of the proposed ideas is founded on the collocation technique (see, e.g., [6]). Let us arrange a dense set of nodes $\left(x_{i}, y_{i}\right), i=1, \ldots, N$, on the contour $L$ : $\left(x_{i}, y_{i}\right) \in L$, for all $i$, which subdivides the contour to $N$ small intervals of the length $\ell_{i}$. 
As follows from the general theory of integral equations of the second kind (see, e.g., [11, Chapter 1]), if $\max _{i}\left(\ell_{i}\right) \rightarrow 0$, then the approximate numerical solution to (2.15) can be obtained by solving the linear algebraic system

$$
\sum_{j=1}^{N} a_{i j} w_{j}=\varphi_{i}^{0}, \quad i=1, \ldots, N,
$$

with

$$
\begin{aligned}
& w_{i}=w\left(x_{i}, y_{i}\right), \quad \varphi_{i}^{0}=-\frac{\sigma_{0}}{2 \pi \mu} \ln \left(x_{i}^{2}+y_{i}^{2}\right), \quad a_{i i}=\frac{1}{2}, \\
& a_{i j}=\frac{1}{2 \pi}\left\{n_{x}\left(x_{j}, y_{j}\right)\left[\frac{x_{i}-x_{j}}{\left(x_{i}-x_{j}\right)^{2}+\left(y_{j}+y_{i}\right)^{2}}+\frac{x_{i}-x_{j}}{\left(x_{i}-x_{j}\right)^{2}+\left(y_{j}-y_{i}\right)^{2}}\right]\right. \\
& \left.\quad-n_{y}\left(x_{j}, y_{j}\right)\left[\frac{y_{j}+y_{i}}{\left(x_{i}-x_{j}\right)^{2}+\left(y_{j}+y_{i}\right)^{2}}+\frac{y_{j}-y_{i}}{\left(x_{i}-x_{j}\right)^{2}+\left(y_{j}-y_{i}\right)^{2}}\right]\right\} \ell_{j}, \quad i \neq j .
\end{aligned}
$$

This system is constructed so that the set of the "inner" discrete integration points $\left\{\left(\xi_{j}, \eta_{j}\right)\right\}$, over which the integration is being performed, coincides with the set of the "outer" nodes $\left\{\left(x_{i}, y_{i}\right)\right\}$, which are used to provide the equality between the left- and the right-hand sides in (2.15). In this case, $\left\{\left(\xi_{j}, \eta_{j}\right)\right\}=\left\{\left(x_{i}, y_{i}\right)\right\}$, and this justifies the method used to be called the "collocation" technique.

Note that the long elements $a_{i j}$, as expressed by the last equality in (3.4), are excluded from the diagonal elements (the case $i=j$ ). These elements correspond to the case when $(\xi, \eta)=(X, Y)$ in the kernel (2.16), and they may have a singular behavior as $(\xi, \eta) \rightarrow(X, Y)$. However, the contribution of such elements to the full sum (2.4) is small (as $\max \left(\ell_{j}\right) \rightarrow 0$ ) when compared with the contribution of the "outer" term in (3.4a) staying outside the integral, which in the discrete form results in the diagonal element $a_{i i}=1 / 2$ in (3.4). Such an approach allows us to avoid unnecessary troubles connected with a potentially singular behavior of the kernel in $(3.4 \mathrm{a})$ as $(\xi, \eta) \rightarrow(X, Y)$.

It should also be noted that another traditional approach to solve arising integral equations, like that studied in the present work, is founded on a choice of a set of basic weighting functions, with further satisfaction of the equation in a weak sense. The collocation technique we prefer to use here does not require any numerical treatment of integrals in order to calculate elements of the matrix for respective linear algebraic system. Our approach can successfully be applied since the real behaviour of the kernel with $(\xi, \eta) \rightarrow(X, Y)$ is continuous - a classical result of the theory of boundary element method for kernels arising from the simple Laplace equation [6]. Moreover, for arbitrary smooth contour, this limiting value is expressed in terms of curvature of the given boundary line $L$.

The problem of void reconstruction from boundary measurements can be formulated in the following way. Let the shape of the deformed boundary surface $(y=0)$, which is caused by the applied point force $\sigma_{0} \delta(x)$, be known on a certain finite-length interval $x \in\left(x^{-}, x^{+}\right)$of this boundary line $y=0$. In this case, we know the function $F_{0}(x)$ in $(3.3)$ 
for $x \in\left(x^{-}, x^{+}\right)$, but we do not know the form of the void, contour $L$. The task is just to reconstruct this contour from the given input data for function $F_{0}(x)$.

Mathematically, the problem can be presented as a (nonlinear) system of two equations (2.15) and (3.3) for the two unknown functions: (1) function $w(\xi, \eta),(\xi, \eta) \in L,(2)$ defining equation of the contour $L$. The considered system seems to be linear with respect to function $w(\xi, \eta)$ but this is so only at first sight, because this function is defined over the contour $L$, which is unknown a priori.

In practice, the measurements on the deformation of the boundary surface cannot be carried out absolutely precisely. This predetermines the input data to be known with a certain error. Therefore, the proposed algorithm should provide a stability with respect to small perturbations of the input data.

\section{Numerical algorithm and examples of reconstruction in the case of round flaw}

The proposed numerical method is founded on the collocation technique described above. For concrete implementation of algebraic system (3.4), we put in the case of smooth contour $L$

$$
\begin{gathered}
\ell_{i}=\frac{\sqrt{\left(x_{i}-x_{i+1}\right)^{2}+\left(y_{i}-y_{i+1}\right)^{2}}+\sqrt{\left(x_{i}-x_{i-1}\right)^{2}+\left(y_{i}-y_{i-1}\right)^{2}}}{2}, \\
n_{x}\left(x_{i}, y_{i}\right)=n_{i}^{x}=\frac{y_{i+1}-y_{i-1}}{\sqrt{\left(x_{i+1}-x_{i-1}\right)^{2}+\left(y_{i+1}-y_{i-1}\right)^{2}}}, \\
n_{y}\left(x_{i}, y_{i}\right)=n_{i}^{y}=\frac{x_{i-1}-x_{i+1}}{\sqrt{\left(x_{i+1}-x_{i-1}\right)^{2}+\left(y_{i+1}-y_{i-1}\right)^{2}}}, \quad 1<i<N, \\
\ell_{N}=\frac{\sqrt{\left(x_{1}-x_{2}\right)^{2}+\left(y_{1}-y_{2}\right)^{2}}+\sqrt{\left(x_{1}-x_{N}\right)^{2}+\left(y_{1}-y_{N}\right)^{2}}}{2}, \\
n_{1}^{x}=\frac{x^{\left(x_{N}-x_{1}\right)^{2}+\left(y_{N}-y_{1}\right)^{2}}+\sqrt{\left(x_{N}-x_{N-1}\right)^{2}+\left(y_{N}-y_{N-1}\right)^{2}}}{2}, \\
\sqrt{\left(x_{2}-x_{N}\right)^{2}+\left(y_{2}-y_{N}\right)^{2}}, \\
y_{1}^{y}=\frac{x_{N}-x_{2}}{\sqrt{\left(x_{2}-x_{N}\right)^{2}+\left(y_{2}-y_{N}\right)^{2}}}, \\
n_{N}^{x}=\frac{y_{1}-y_{N-1}}{\sqrt{\left(x_{1}-x_{N-1}\right)^{2}+\left(y_{1}-y_{N-1}\right)^{2}}}, \\
x_{N-1}-x_{1}
\end{gathered}
$$

Such a construction automatically provides a symmetric solution for symmetric contours. 
These formulas are valid for arbitrary smooth contour $L$. However, if the flaw is a round cylinder of the radius $a$, with its center being located at the point $(c, h)$, then the above formulas can be written in a more concrete form since

$$
x_{i}=c+a \cos \left(\beta_{i}\right), \quad y_{i}=h+a \sin \left(\beta_{i}\right), \quad \beta_{i}=\varepsilon(i-0.5), \quad \varepsilon=\frac{2 \pi}{N} .
$$

Under such conditions the reconstruction problem becomes 3-dimensional, in the sense that this consists of a search of the three parameters $h, c, a$. Two different algorithms can be proposed to resolve this inverse problem.

The first one operates with the set of $N+3$ unknown quantities $h, c, a$, and $w_{i}(i=$ $1, \ldots, N)$ from the system (3.4) ( $N$ equations), which has to be considered after substitution of (4.2) into (3.4). The additional set of required equations may be constructed by choosing a number of nodes $x_{m} \in\left(x^{-}, x^{+}\right)(m=1, \ldots, M)$ in the equality (3.3) over the interval where the input data of the measured values $F_{0}\left(x_{m}\right)$ is collected. If we take $M>3$, then we come to an overdetermined system of $N+M$ relations for $N+3$ unknown quantities.

In order to resolve this system of equations, we can pose a problem on minimization of the discrepancy functional:

$$
\begin{gathered}
\min \left[\Omega_{1}\left(h, c, a,\left\{w_{i}\right\}\right)\right], \\
\Omega_{1}\left(h, c, a,\left\{w_{i}\right\}\right)=\left\|\sum_{j=1}^{N} a_{i j} w_{j}-\varphi_{i}^{0}\right\|^{2}+\left\|\frac{1}{\pi} \sum_{j=1}^{N} \frac{\left(x_{m}-\xi_{j}\right) n_{j}^{x}-\eta_{j} n_{j}^{y}}{\left(x_{m}-\xi_{j}\right)^{2}+\eta_{j}^{2}} w_{j} \ell_{j}-F_{0}\left(x_{m}\right)\right\|^{2} \\
=\sum_{i=1}^{N}\left(\sum_{j=1}^{N} a_{i j} w_{j}-\varphi_{i}^{0}\right)^{2}+\frac{1}{\pi^{2}} \sum_{m=1}^{M}\left[\sum_{j=1}^{N} \frac{\left(x_{m}-\xi_{j}\right) n_{j}^{x}-\eta_{j} n_{j}^{y}}{\left(x_{m}-\xi_{j}\right)^{2}+\eta_{j}^{2}} w_{j} \ell_{j}-F_{0}\left(x_{m}\right)\right]^{2} .
\end{gathered}
$$

In the case when the input data is given exactly, this functional attains the minimum $\min \left(\Omega_{1}\right)=0$ just on the exact contour. The question whether there is another combination of the sought quantities, which provides the zero value of the functional $\Omega_{1}$, is related to the uniqueness of the solution to the formulated reconstruction problem. This question is out of the present study, whose principal goal is to propose an efficient concrete numerical algorithm of the reconstruction and to test it in the case of round flaws rather than to investigate (also important in some aspects) questions about solvability and uniqueness.

Another approach can be founded on explicit (numerical) resolution of system (3.4) considered as a linear algebraic system only for the values of $w_{i}$. If this system is represented in the operator form as

$$
A w=\varphi^{0}, \quad A=\left(a_{i j}\right), w=\left(w_{i}\right), \varphi^{0}=\left(\varphi_{i}^{0}\right),(i, j=1, \ldots, N)
$$

then its inversion is

$$
w=A^{-1} \varphi^{0} \Longrightarrow w_{i}=\left(A^{-1} \varphi^{0}\right)_{i}
$$


Obviously, operator $A^{-1}$ depends on the three parameters: $A^{-1}=A^{-1}(h, c, a)$, so the substitution of (4.5) into (3.3) results, in the discrete form, in the overdetermined system of nonlinear equations for parameters $h, c, a$ :

$$
\frac{1}{\pi} \sum_{j=1}^{N} \frac{\left(x_{m}-\xi_{j}\right) n_{j}^{x}-\eta_{j} n_{j}^{y}}{\left(x_{m}-\xi_{j}\right)^{2}+\eta_{j}^{2}}\left[A^{-1}(h, c, a) \varphi^{0}\right]_{j} \ell_{j}=F_{0}\left(x_{m}\right), \quad m=1, \ldots, M
$$

This can also be resolved by a minimization of the discrepancy functional:

$$
\begin{gathered}
\min \left[\Omega_{2}(h, c, a)\right], \\
\Omega_{2}(h, c, a)=\left\|\frac{1}{\pi} \sum_{j=1}^{N} \frac{\left(x_{m}-\xi_{j}\right) n_{j}^{x}-\eta_{j} n_{j}^{y}}{\left(x_{m}-\xi_{j}\right)^{2}+\eta_{j}^{2}}\left[A^{-1}(h, c, a) \varphi^{0}\right]_{j} \ell_{j}-F_{0}\left(x_{m}\right)\right\|^{2} \\
=\frac{1}{\pi^{2}} \sum_{m=1}^{M}\left\{\sum_{j=1}^{N} \frac{\left(x_{m}-\xi_{j}\right) n_{j}^{x}-\eta_{j} n_{j}^{y}}{\left(x_{m}-\xi_{j}\right)^{2}+\eta_{j}^{2}}\left[A^{-1}(h, c, a) \varphi^{0}\right]_{j} \ell_{j}-F_{0}\left(x_{m}\right)\right\}^{2} .
\end{gathered}
$$

By the same reason as in the minimization of functional $\Omega_{1},(4.3)$, in the case of exact input data, a zero minimum of $\Omega_{2}$ corresponds to the exact solution of the inverse problem under consideration. And we are not able to predict a priori whether this solution is unique.

By their basic ideas and in some technical aspects, these approaches are different. The principal difference is that the first algorithm does not require any solution of the direct problem, as it is formulated in the form of integral equation (2.15), or in the equivalent discrete form of linear algebraic system (3.4).

Another difference between the minimization of the two functionals, $\Omega_{1}$ and $\Omega_{2}$, is that the latter is really a usual function of three variables. From this point of view the minimization of $\Omega_{2}$ seems to be a simpler problem.

However, any regular method to solve both minimization problems is based on a calculation of the gradient operator of the corresponding functional. For $\Omega_{1}$, this can be found analytically in an explicit form since the dependence of the functional upon $w_{i}$ is quadratic and explicit, and the dependence on the parameters $(h, c, a)$ is more complex but still explicit (in fact, $a_{i j}$ is explicitly written in (3.4b) in terms of $\left(x_{i}, y_{i}\right)$, and the latter clearly contains the three parameters from (4.2)). From this point of view, the determination of the gradient is a simpler task just in the case of $\Omega_{1}$, since for this functional the gradient can be easily calculated analytically. For the functional $\Omega_{2}$, this gradient can be calculated numerically.

As soon as the method to calculate the gradient of $\Omega_{1}$ and $\Omega_{2}$ has been arranged, the minimization of these functionals can be achieved by some iterative gradient process, like steepest descent method or conjugate gradient method (see, e.g., [9]). Both iterative methods converge to the exact solution in the case of linear operator equation, which corresponds to a quadratic functional. Our inverse problem is nonlinear, so we cannot 
Table 4.1

\begin{tabular}{ccccc}
\hline Input data error & $h$ & $c$ & $a$ & Type of result \\
\hline \multirow{2}{*}{$0 \%$} & 3.000 & 0.000 & 1.000 & Exact \\
& 3.044 & 0.001 & 0.993 & Restored \\
& $1.47 \%$ & - & $0.7 \%$ & Relative error \\
\hline \multirow{2}{*}{$0 \%$} & 1.500 & 2.000 & 1.000 & Exact \\
& 1.508 & 2.002 & 1.003 & Restored \\
& $0.53 \%$ & $0.1 \%$ & $0.3 \%$ & Relative error \\
\hline \multirow{2}{*}{$0 \%$} & 7.000 & 4.000 & 5.000 & Exact \\
& 6.977 & 3.970 & 4.983 & Restored \\
& $0.33 \%$ & $0.75 \%$ & $0.34 \%$ & Relative error \\
\hline \multirow{2}{*}{$0 \%$} & 2.000 & -5.000 & 0.300 & Exact \\
& 2.007 & -4.980 & 0.300 & Restored \\
& $0.35 \%$ & $0.4 \%$ & $0 \%$ & Relative error \\
\hline
\end{tabular}

prove strictly that the iterative scheme converges. However, since any smooth functional is locally quadratic near a minimum, there is a good chance that this iterative method is convergent.

The most important point is that any iterative method provides a convergence to a local minimum only. In the case of nonlinear equations, such values of local minima may be too far from the desired value $\Omega_{1,2}=0$.

For this reason, we used in our numerical experiments a version of the method of random search contiguous to the one described in detail in [7]. This provides a search of the global minima by moving step by step among the most promising iterations. When performing the numerical implementation, we could clearly observe that the minimization of $\Omega_{1}$ and $\Omega_{2}$ gives results very close to each other, but operation with $\Omega_{2}$ permits reconstruction within a shorter time of computations.

Some examples of the reconstruction are demonstrated in Table 4.1. For all examples demonstrated below, we used $M=200$ points of measurements over the interval $x \in$ $(-5,5)$ to form the array of the input data, so that $x_{m}=0.05 m, x_{m+100}=-0.05 m, m=$ $1, \ldots, M / 2$. It is clear that with such a choice of the trial points they represent a relatively uniform set around the applied force.

These results are related to the case of exact input data. The latter was obtained by a solution of the direct boundary value problem by boundary element technique. The physical conclusions on Table 4.1 are quite evident.

Then, we studied the stability of the proposed algorithm if the input data is given with an error. In order to model the input data with a certain error, we first did construct the solution of respective direct problem, and then arranged some stochastic perturbations of the so-obtained data. Some results of such a numerical simulation are shown in Table 4.2.

Further increase in the error of the input data leads to Table 4.3.

From the presented results of the numerical simulation, as well as from other numerous calculations performed, the authors come to some important conclusions. 
Table 4.2

\begin{tabular}{ccccc}
\hline Input data error & $h$ & $c$ & $a$ & Type of result \\
\hline \multirow{3}{*}{$10 \%$} & 1.000 & 0.000 & 0.500 & Exact \\
& 1.013 & -0.004 & 0.512 & Restored \\
& $1.3 \%$ & - & $2.4 \%$ & Relative error \\
\hline \multirow{3}{*}{$10 \%$} & 4.000 & -2.500 & 1.500 & Exact \\
& 4.019 & -2.524 & 1.502 & Restored \\
& $0.47 \%$ & $0.96 \%$ & $0.13 \%$ & Relative error \\
\hline \multirow{2}{*}{$10 \%$} & 8.000 & 5.000 & 0.100 & Exact \\
& 7.485 & 4.259 & 0.096 & Restored \\
& $6.44 \%$ & $14.8 \%$ & $4 \%$ & Relative error \\
\hline \multirow{3}{*}{$10 \%$} & 8.000 & 5.000 & 1.200 & Exact \\
& 8.010 & 4.937 & 1.197 & Restored \\
& $0.12 \%$ & $1.26 \%$ & $0.25 \%$ & Relative error \\
\hline
\end{tabular}

Table 4.3

\begin{tabular}{ccccc}
\hline Input data error & $h$ & $c$ & $a$ & Type of result \\
\hline \multirow{2}{*}{$20 \%$} & 3.000 & 2.500 & 2.000 & Exact \\
& 2.998 & 2.498 & 2.002 & Restored \\
& $0.07 \%$ & $0.08 \%$ & $0.1 \%$ & Relative error \\
\hline \multirow{2}{*}{$20 \%$} & 7.000 & -2.500 & 0.600 & Exact \\
& 6.978 & -2.418 & 0.597 & Restored \\
& $0.31 \%$ & $3.28 \%$ & $0.5 \%$ & Relative error \\
\hline \multirow{2}{*}{$20 \%$} & 8.000 & 0.000 & 4.000 & Exact \\
& 7.858 & 0.054 & 3.951 & Restored \\
& $1.77 \%$ & - & $1.22 \%$ & Relative error \\
\hline \multirow{2}{*}{$20 \%$} & 0.500 & -4.500 & 0.200 & Exact \\
& 0.521 & -4.537 & 0.202 & Restored \\
& $4.2 \%$ & $0.82 \%$ & $1 \%$ & Relative error \\
\hline
\end{tabular}

(1) It is interesting to notice that Table 4.3 in some cases shows better results than Table 4.2, despite rougher input data. This indicates an important property that the precision of the reconstruction is less dependent on the error of the input data than on the geometry of the void, that is, its size and position.

(2) The most critical parameter seems to be the ratio of the radius to the depth, as can be seen from the third line of Table 4.2. This result is not unexpected and can be explained by observing that the decrease of the flaw size and the increase of the depth reduce the amplitude of the free surface deformation.

(3) The most sensitive parameter in view of relative error is the horizontal position of the void. This can be observed from the relative error lines reported on the tables. 
(4) The results do not depend significantly on the precision of the input data. This may be connected with the overdetermined character of the input data for the inverse problem. It also indicates implicitly that the input data randomly perturbed have the same mean value as precise input data. In other words, the proposed algorithm arranges automatically something like a filtering of a randomly distributed error.

One of the authors' next works will be devoted to reconstruction of voids of a more complex geometry located in the elastic medium.

\section{Acknowledgments}

The present work was supported in part by the Program on Russian Leading Scientific Schools in Mechanics (Grant 2113.2003.1). The paper has also been supported by the Italian Ministero dell'Istruzione, dell'Università e della Ricerca (MIUR) through its national and local (60\%) projects.

\section{References}

[1] G. Alessandrini, E. Beretta, and S. Vessella, Determining linear cracks by boundary measurements: Lipschitz stability, SIAM J. Math. Anal. 27 (1996), no. 2, 361-375.

[2] S. Andrieux and A. Ben Abda, Identification of planar cracks by complete overdetermined data: inversion formulae, Inverse Problems 12 (1996), no. 5, 553-563.

[3] T. Bannour, A. Ben Abda, and M. Jaoua, A semi-explicit algorithm for the reconstruction of $3 D$ planar cracks, Inverse Problems 13 (1997), no. 4, 899-917.

[4] J. R. Barber, Elasticity, 2nd ed., Solid Mechanics and Its Applications, vol. 107, Kluwer Academic, Dordrecht, 2002.

[5] A. Ben Abda, M. Kallel, J. Leblond, and J.-P. Marmorat, Line segment crack recovery from incomplete boundary data, Inverse Problems 18 (2002), no. 4, 1057-1077.

[6] M. Bonnet, Boundary Integral Equations Methods for Solids and Fluids, John Wiley \& Sons, New York, 1999.

[7] A. Corana, M. Marchesi, C. Martini, and S. Ridella, Minimizing multimodal functions of continuous variables with the "simulated annealing" algorithm, ACM Trans. Math. Software 13 (1987), no. 3, 262-280.

[8] A. Friedman and M. Vogelius, Determining cracks by boundary measurements, Indiana Univ. Math. J. 38 (1989), no. 3, 527-556.

[9] P. E. Gill, W. Murray, and M. H. Wright, Practical Optimization, Academic Press, London, 1981.

[10] O. D. Kellogg, Foundations of Potential Theory, Dover, New York, 1953.

[11] M. A. Sumbatyan and A. Scalia, Equations of Mathematical Diffraction Theory, Differential and Integral Equations and Their Applications, vol. 5, Chapman \& Hall/CRC, Florida, 2005.

Mezhlum A. Sumbatyan: Faculty of Mechanics and Mathematics, Rostov State University,

5 Zorge Street, Rostov-on-Don 344090, Russia

E-mail address: sumbat@math.rsu.ru

Vincenzo Tibullo: Dipartimento di Ingegneria dell' Informazione e Matematica Applicata, University of Salerno, Via Ponte don Melillo, 84084 Fisciano (SA), Italy

E-mail address: tibullo@diima.unisa.it

Vittorio Zampoli: Dipartimento di Ingegneria dell' Informazione e Matematica Applicata, University of Salerno, Via Ponte don Melillo, 84084 Fisciano (SA), Italy

E-mail address: zampoli@diima.unisa.it 


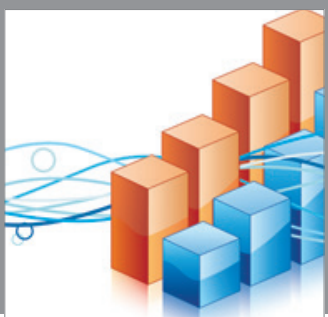

Advances in

Operations Research

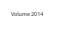

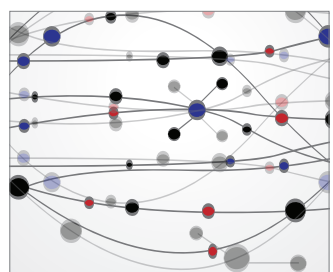

\section{The Scientific} World Journal
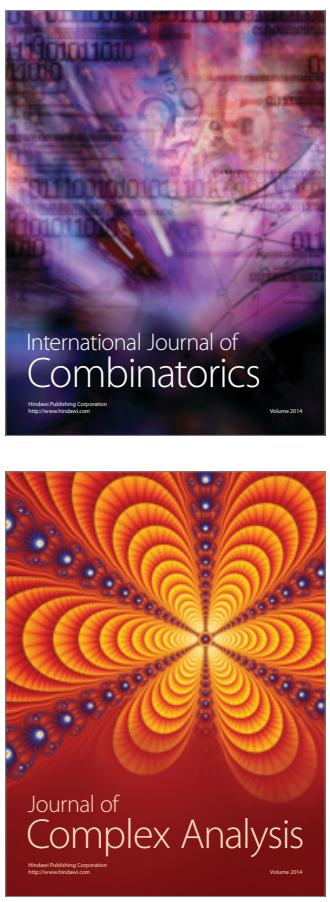

International Journal of

Mathematics and

Mathematical

Sciences
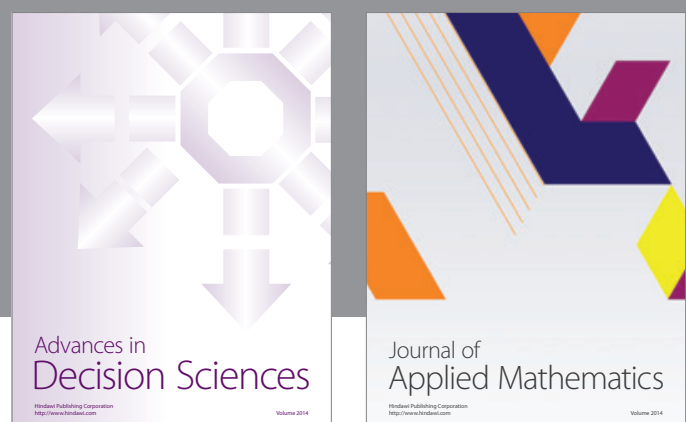

Journal of

Applied Mathematics
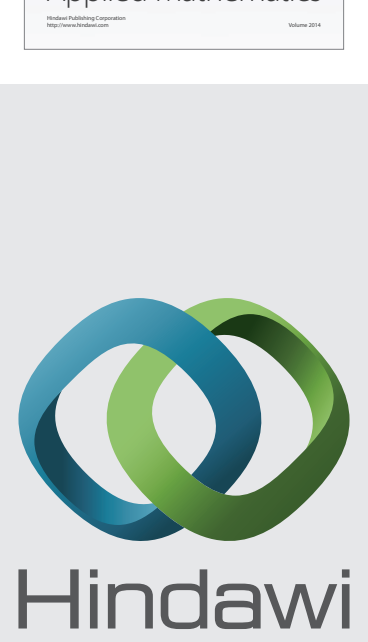

Submit your manuscripts at http://www.hindawi.com
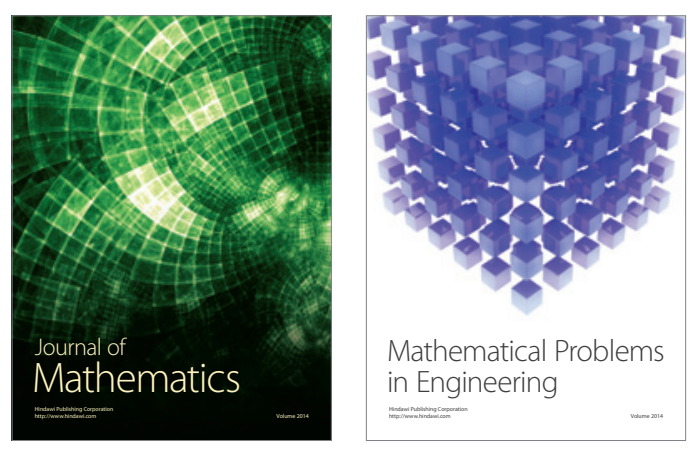

Mathematical Problems in Engineering
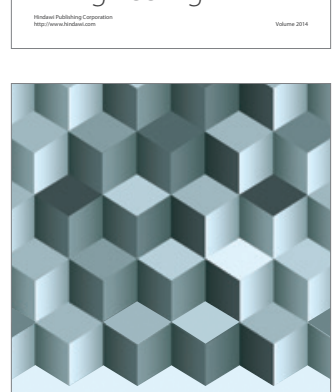

Journal of

Function Spaces
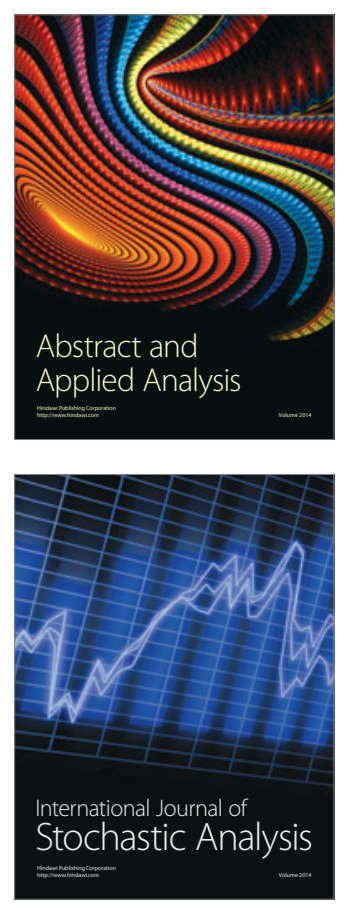

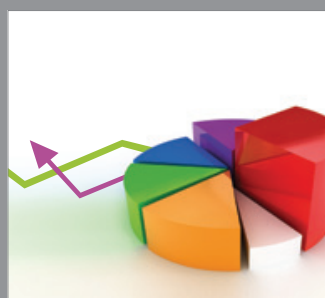

ournal of

Probability and Statistics

Promensencen
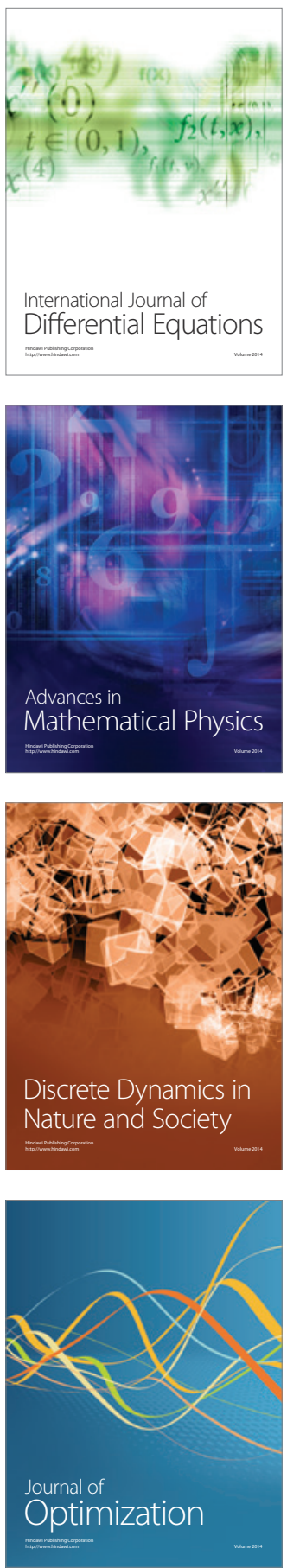